\title{
A COMPACT SEMILATTICE ON THE HILBERT CUBE WITH NO INTERVAL HOMOMORPHISM
}

\author{
GERHARD GIERZ
}

(Communicated by Doug W. Curtis)

\begin{abstract}
An example for a compact semilattice with no interval homomorphism is given. The underlying space of the semilattice is homeomorphic to the Hilbert cube.
\end{abstract}

Introduction. A compact semilattice $S$ is a compact, idempotent, commutative topological semigroup with identity. The standard example of such a compact semilattice is the unit interval $[0,1]$ under the operation $x y=\max \{x, y\}$. It is an old problem to decide whether a compact semilattice allows an embedding into a power of $[0,1]$, i.e. whether there are enough continuous semilattice homomorphisms into the unit interval to separate points. Jimmie D. Lawson [4] constructed the first two examples of compact semilattices with no nontrivial continuous homomorphisms into $[0,1]$. Up to today, only one other such example is known, and this example is derived from a compact convex set without extreme points. The constructions for those examples are fairly complicated.

Thus it became an interesting question to find topological restrictions which force a compact semilattice to allow enough semilattice homomorphisms into the unit interval to separate the points. Again Jimmie Lawson [3] has shown that a topological semilattice on a finite dimensional Peano continuum or on a compact totally disconnected space must have this property. His two counterexamples live on a one-dimensional metric continuum and on an infinite dimensional Peano continuum, respectively.

In this note, I shall give an easy construction of a compact semilattice $L$ without any nontrivial homomorphisms into the unit interval. As a topological space, $L$ will be homeomorphic to the Hilbert cube. Furthermore, $L$ has the interesting property that is order generated by its irreducible elements.

The construction. Let $x=\left(x_{n}\right)_{n}$ and $y=\left(y_{n}\right)_{n} \in[0,1]^{\mathbf{N}}$ be two sequences. We define a new sequence $x \vee y$ by $(x \vee y)_{n}=\max \left\{x_{n}, y_{n}\right\}$. Under the operation $\vee$, the set $[0,1]^{\mathbf{N}}$ is a compact semilattice. For every positive integer $n$ define a mapping $f_{n}:[0,1]^{\mathbf{N}} \rightarrow[0,1]$ by

$$
f_{n}\left(x_{1}, x_{2}, x_{3}, \ldots\right)=\ln \left(1+\sum_{i=1}^{n} x_{i}\right) / \ln (1+n) .
$$

Clearly, each $f_{n}$ is monotone increasing and continuous. Furthermore, we have

$$
f_{n}(x \vee y) \leq \max \left\{f_{n}(x), f_{n}(y)\right\}+\ln 2 / \ln (n+1),
$$

Received by the editors August 22, 1986.

1980 Mathematics Subject Classification (1985 Revision). Primary 06B30, 06F30, 54H12.

Key words and phrases. Compact semilattices, interval homomorphisms. 
i.e. the mappings $f_{n}$ are almost semilattice homomorphisms. Indeed, assume that $x_{1}+\cdots+x_{n} \leq y_{1}+\cdots+y_{n}$. Then

$$
\begin{aligned}
\ln (1 & \left.+\max \left\{x_{1}, y_{1}\right\}+\cdots+\max \left\{x_{n}, y_{n}\right\}\right) \\
& \leq \ln \left(2+\left(x_{1}+y_{2}\right)+\cdots+\left(x_{n}+y_{n}\right)\right) \\
& \leq \ln \left(2\left[1+y_{1}+\cdots+y_{n}\right]\right) \\
& =\max \left\{\ln \left(1+x_{1}+\cdots+x_{n}\right), \ln \left(1+y_{1}+\cdots+y_{n}\right)\right\}+\ln 2 .
\end{aligned}
$$

Define

$$
f:[0,1]^{\mathbf{N}} \rightarrow[0,1], \quad z \mapsto \sup _{n \in N} f_{n}(x)
$$

It follows from [1, VI.4.1, VI.4.2] that the set

$$
S=\left\{(x, r) \in[0,1]^{\mathbf{N}} \times[0,1]: f(x) \leq r\right\}
$$

is a closed subset of $[0,1]^{\mathbf{N}} \times[0,1]$ and a compact semilattice under the operation $(x, r) \cdot(y, s)=(x \vee y, \max \{r, s, f(x \vee y)\})$. Let $z \in[0,1]^{\mathbf{N}}$ be the element with all coordinates 0 , and let $\phi: S \rightarrow[0,1]$ be an arbitrary continuous semilattice homomorphism. We show:

$$
\phi(z, 0)=\phi(z, 1)
$$

Suppose not. Then $\phi(x, 0)<\phi(z, 1)$; w.l.o.g. we may assume that $\phi(z, 0)=0$ and $\phi(z, 1)=1$. The set $U=\{x, s) \in S: \phi(x, s)<1 / 2\}$ would be an open neighborhood of $(z, 0)$ closed under the semilattice operation and not containing $(z, 1)$ in its closure. Let $e_{n} \in[0,1]^{\mathbf{N}}$ be the $n$th unit vector. Then $\alpha_{n}=\left(e_{n}, \ln 2 / \ln (n+1)\right) \in S$. Since $\lim _{n \rightarrow \infty} \alpha_{n}=(z, 0)$ there is an integer $N$ such that $\alpha_{n} \in U$ for all $n \geq 0$. Let $u_{n} \in[0,1]^{\mathbf{N}}$ be the vector having 0's in the first $n-1$ coordinates and 1's in all the others. Clearly $f\left(u_{n}\right)=1$ for all $n$. Since $\left(u_{m}, 1\right)=\lim _{k \rightarrow \infty} \alpha_{m} \cdots \alpha_{m+k}$, we conclude that $\left(u_{m}, 1\right) \in \bar{U}$ for every $m>N$, and since $\lim _{m \rightarrow \infty}\left(u_{m}, 1\right)=(z, 1)$, we arrive at the contradiction $(z, 1) \in \bar{U}$.

Consider the (closed) equivalence relation $\Theta$ on $S$ which has $I=\{(x, 1) \in$ $\left.S: x \in[0,1]^{\mathbf{N}}\right\}$ as its only nontrivial equivalence class. Then $I$ is an ideal of the semigroup $S$, and the Rees quotient $S / I=S / \Theta$ carries again in canonical way the structure of a compact semilattice. Now (2) implies that for every continuous semilattice homomorphism $\psi: S / I \rightarrow[0,1]$ we have $\psi(I)=\psi\{(z, 0)\}$. Since in $S / I$ the elements $I$ and $\{(z, 0)\}$ act as zero element and identity, respectively, it follows that every continuous semilattice homomorphism $\phi: S / I \rightarrow[0,1]$ is constant.

It remains to show that as a topological space, $S / I$ is homeomorphic to the Hilbert cube. Firstly, we verify that $S$ is homeomorphic to the Hilbert cube. For every positive integer $N$, let

$$
\rho_{n}:\left[-n \ln 2, \ln \left(1+1 / 2^{n}\right)\right] \rightarrow[0,1], \quad x \mapsto e^{x}-1 / 2^{n}
$$

Clearly, each $\rho_{n}$ is a homeomorphism, and therefore the map

$$
\rho: \prod_{1 \leq n}\left[-n \ln 2, \ln \left(1+1 / 2^{n}\right)\right] \rightarrow[0,1]^{\mathbf{N}}, \quad\left(x_{n}\right)_{n} \mapsto\left(\rho_{n}\left(x_{n}\right)\right)_{n},
$$

is also a homeomorphism. For each $n$ we have

$$
f_{n} \circ \rho\left(x_{1}, x_{2}, \ldots\right)=\ln \left(1 / 2^{n+1}+e^{x_{1}}+\cdots+e^{x_{n}}\right) / \ln (1+n),
$$


and it follows from the Minkowski inequality that this is a convex function. Hence $(\rho \times \mathrm{id})^{-1}(S)$ is a convex set, i.e. $S$ is homeomorphic to an infinite dimensional compact convex subset of Hilbert space. It follows now from a classical result of O. H. Keller [2] that $S$ is homeomorphic to the Hilbert cube. Finally, we obtain that $(\rho \times \mathrm{id})^{-1}(I)$ is a face of the compact convex set $(\rho \times \mathrm{id})^{-1}(S)$. It now follows easily from Hilbert cube theory that $S / I$ is homeomorphic to the Hilbert cube.

\section{REFERENCES}

1. G. Gierz, et al., A compendium of continuous lattices, Springer-Verlag, Berlin and New York, 1980.

2. O. H. Keller, Die Homoimorphie der kompakten konvexen Mengen im Hilbertschen Raum, Math. Ann. 105 (1981), 748-758.

3. J. D. Lawson, Topological semilattices with small semilattices, J. London Math. Soc. 1 (1969), 719-724.

4. __ Lattices with no interval homomorphisms, Pacific J. Math. 32 (1970), 459-465.

DEPARTMENT OF MATHEMATICS, UNIVERSity OF CALIFORNiA AT RIVERSIDE, RIVERSIDE, CALIFORNIA 92521 